\title{
Measurement of photoluminescence spectral linewidth of a GaAs quantum well in perpendicular electric fields: Evidence of a crossover from trions to an electron-hole gas
}

\author{
M. Yamaguchi, ${ }^{1}$ S. Nomura, ${ }^{2}$ H. Tamura, ${ }^{1}$ and T. Akazaki ${ }^{1}$ \\ ${ }^{1}$ NTT Basic Research Laboratories, NTT Corporation, 3-1 Morinosato-Wakamiya, Atsugi-shi, Kanagawa 243-0198, Japan \\ ${ }^{2}$ Division of Physics, University of Tsukuba, 1-1-1 Tennodai Tsukuba, Ibaraki 305-8571, Japan \\ (Received 2 October 2012; revised manuscript received 19 December 2012; published 25 February 2013)
}

\begin{abstract}
By measuring the photoluminescence linewidth of a GaAs quantum well under perpendicular electric fields, we have traced the variation of the effective radius $a^{*}$ of a charged exciton (trion) as a function of electron density. The $a^{*}$ increases sharply above a critical density $n_{s}=2 \times 10^{14} \mathrm{~m}^{-2}$, which is consistent with the decrease of the screening length predicted by nonlinear-screening theory. Our analysis shows that the crossover from trions to the two-dimensional electron gas plus hole is generated by Coulomb screening in the high-electron-density regime, eliminating the possible mechanism by the trion localization effect due to the single-electron localization.
\end{abstract}

DOI: 10.1103/PhysRevB.87.081310

PACS number(s): 78.67.De, 78.20.Jq, 78.55.Cr

The electron-hole system in semiconductors has been providing intriguing subjects for study in the field of condensed matter physics for years. Interactions among electrons and holes generate various interesting phenomena, such as the exciton-Mott transition, ${ }^{1}$ that have attracted theoretical and experimental interest. ${ }^{2-6}$ A charged exciton (or trion), ${ }^{7}$ a bound state of two electrons and a hole mediated by the Coulomb interaction, is the most stable state in the charge-imbalanced system formed in a semiconductor quantum well (QW) ${ }^{8}$ In the high-electron-density regime, the system is regarded as a twodimensional electron gas plus a hole (2DEG-h) state, which has also been extensively studied for decades. ${ }^{9}$ The crossover between trions and 2DEG-h states has been theoretically predicted, ${ }^{10}$ although the role of disorder in the crossover was not clarified. Experimentally, the crossover between trions and the 2DEG-h state in modulation-doped GaAs QWs has been reported, ${ }^{11,12}$ where the crossover was characterized as an abrupt change in the spectrum as the electron density was modulated. A near-field optical measurement using a modulation-doped sample with a spacer width of $37.5 \mathrm{~nm}$ directly confirmed that there were no large clusters of electrons and the electrons are singly localized in separated potential valleys. ${ }^{11}$ Yusa et al. ${ }^{12}$ showed a sharp onset of the excitonic absorption at the critical electron density $n_{c}=2.5 \times 10^{14} \mathrm{~m}^{-2}$ and pointed out the role of electrostatic potential fluctuation. The electron-density regime where the crossover of the bound to unbound states occurs often overlaps the electron-density regime for the localized-delocalized transition.

To examine the intrinsic mechanism of the crossover, it is desirable to separate the crossover and the electron localization regimes. A gated undoped GaAs QW sample is suitable for this purpose since the length scale of the potential fluctuation is significantly larger than that of a modulationdoped QW sample. This enables us to investigate the crossover between trions and 2DEG-h states separated from the electron localization effect. Actually, in a previous paper, ${ }^{13}$ photoluminescence (PL) spectra with changing electron density clearly identified three distinct regimes, i.e., the single-electronlocalization regime, the nonlinear-screening regime, and the linear-screening regime. In this Rapid Communication, we show that the crossover from trions to the 2DEG-h occurs in the nonlinear-screening regime at a density much higher than the critical density for the single-electron delocalization. Our observation unambiguously reveals that the crossover is generated purely by the variation of screening length of the Coulomb interaction between electrons and a hole, irrelevant to trion localization due to the single-electron localization.

We used a molecular-beam-epitaxy grown 20-nm GaAs QW sandwiched by undoped $\mathrm{Al}_{0.2} \mathrm{Ga}_{0.8}$ As barriers as shown in Fig. 1(a). A semitransparent Ti/Au film of $15-\mathrm{nm}$ total thickness deposited on the surface and a heavily $n$-doped GaAs wafer were used as front and back gates, which were separated by $250 \mathrm{~nm}$ and $620 \mathrm{~nm}$ from the QW, respectively. AuGeNi was alloyed to provide an ohmic contact to the QW. ${ }^{14}$ The electron density and electric field can be controlled independently by tuning the front- and back-gate voltages. Due to the large distance between the surface and the QW, the electrostatic potential fluctuation has a remarkably large length scale of $250 \mathrm{~nm}$, which is much larger than the trion effective radius $a_{0}^{*} . a_{0}^{*}$ has been derived to be $12^{15}$ and $24 \mathrm{~nm}^{16}$ for 20 -nm GaAs QW by using different definitions. The PL measurements were performed at $100 \mathrm{mK}$ in a dilution refrigerator. The laser light was introduced to the sample surface through an optical fiber at an excitation power density of $<1 \mathrm{~mW} / \mathrm{cm}^{2}$ at $800.0 \mathrm{~nm}(1.5498 \mathrm{eV})$. In this condition, the trion density is much smaller than the electron density and we can neglect the interaction between photoexcited particles, such as the triontrion interaction. The PL was collected with the same fiber and dispersed with a 1-m monochromator and detected by a liquid nitrogen-cooled charge-coupled device. The electron density was estimated from the PL anomaly at the integer quantum Hall state $v=1$ in several magnetic fields (see Ref. 17 for details) by fitting to a linear function of $V_{b}$ and $V_{f}{ }^{18}$ given by the equation $n_{e}=0.99 \times 10^{15}\left[V_{b}-\left(1.89-2.65 V_{f}\right)\right]$. We independently measured the capacitance between the back gate and the QW, and the estimated electron density was in good agreement with the above equation. The above linear function can be extrapolated to lower density, ${ }^{17}$ and the electron density possibly deviates from the above equation only below $1.0 \times 10^{14} \mathrm{~m}^{-2}$ because of residual charges supplied from the barrier layers.

Before describing the experimental results, we consider how potential fluctuations affect the electron states as the electron density is changed. As originally discussed by Efros, ${ }^{19}$ 

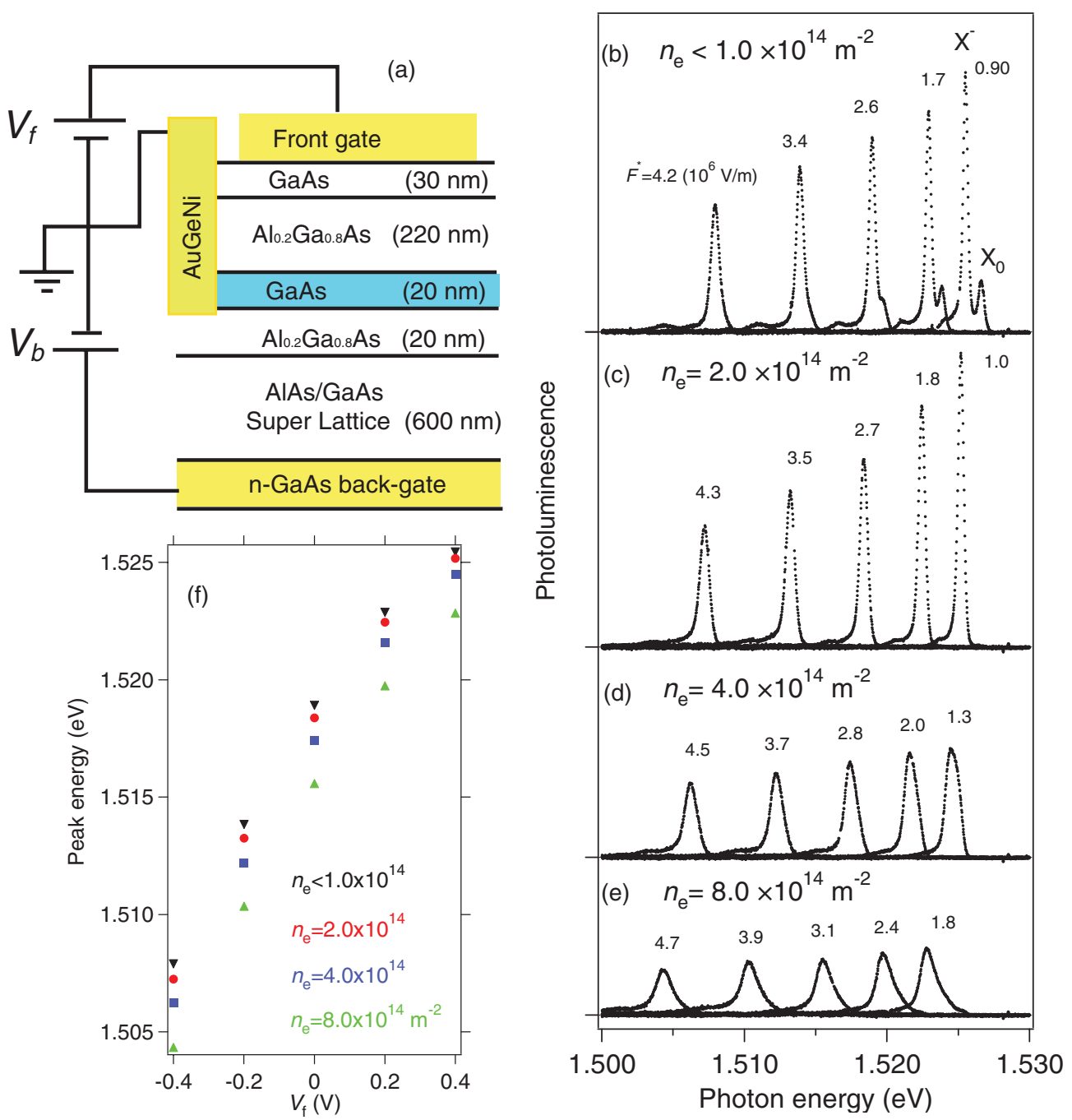

FIG. 1. (Color online) (a) Schematic of the layer structure of the sample. (b)-(e) PL spectra for different electric fields at $n_{e}<1.0 \times 10^{14} \mathrm{~m}^{-2}$ and $n_{e}=2.0,4.0,8.0 \times 10^{14} \mathrm{~m}^{-2}$. A common base curve due to the emission from the substrate was subtracted. Electric fields $F^{*}$ are evaluated from the shift of PL emission energy (see text). (f) The PL peak emission energy as a function of $V_{f}$.

electrons are induced at the semiconductor heterointerface inhomogenously at low electron density because of the existence of the electrostatic potential fluctuations $V_{\text {remote }}$ originating from the remote ion layer. $V_{\text {remote }}$ is characterized by two parameters: the spatial length scale $s$ and the charge density $n_{i}$ of the remote ion layer. In our sample, $s=250 \mathrm{~nm}$ and $n_{i}=2.0 \times 10^{16} \mathrm{~m}^{-2}$ were estimated from the geometry and the position of the capacitance peak, respectively. ${ }^{13}$ The phase of electron states in the electrostatic disorder potential is separated by three critical electron densities: the single-electron localization density $n_{\text {sel }}=1 / s^{2}$, the percolation density $n_{p}=0.12 \sqrt{n_{i}} / s$, and the linear screening density $n_{\mathrm{ls}}=0.5 \sqrt{n_{i}} / s{ }^{20} n_{\text {sel }}$ represents the maximum density at which the single electron can occupy the individual potential valleys. $n_{p}$ corresponds to the density at which the electron puddles percolate in the two-dimensional (2D) plane and where half of the $2 \mathrm{D}$ plane is covered with electrons. Above $n_{\mathrm{ls}}$, the entire area of the 2D plane is covered with electrons. Note that, if $s^{-1} \gg 0.12 \sqrt{n_{i}}$, the electron state transits directly from the single-electron localization to high-density 2DEG. This is the case for a modulation-doped GaAs QW with a short spacer width. In our sample, we estimate $n_{\text {sel }}=2 \times 10^{13} \mathrm{~m}^{-2}$, $n_{p}=6.8 \times 10^{13} \mathrm{~m}^{-2}$, and $n_{\mathrm{ls}}=2.8 \times 10^{14} \mathrm{~m}^{-2}$. Then the nonlinear screening regime with the electron puddle states is expected to emerge as electron density changes, where the crossover of the excitonic bound state to the unbound 2DEG-h state is expected to occur.

In discussing the spatial randomness, three kinds of disorder source are to be considered. ${ }^{21,22}$ One is the electrostatic random potential $V_{\text {remote }}$ as mentioned above. Although $V_{\text {remote }}$ affects the 2DEG state and depends strongly on the electron density, the contribution of $V_{\text {remote }}$ to the PL emission energy is small in our sample because the spatial length scale of this fluctuation $s(=250 \mathrm{~nm})$ is much larger than the $X^{-}$radius and the random energy shift cancels out in the emission energy after the electron-hole recombination. As a second source, it has been reported recently that the potential due to background impurities in the barrier layer plays an important role in trion localization. $^{22}$ The third source of disorder potential is the monolayer fluctuation of the QW width, which directly affects 
the $X^{-}$emission energy. This energy shift $W^{(i)}$ in the QW is written by

$$
W^{(i)}=\Phi^{(i)}+E_{\mathrm{QCSE}}^{(i)} \quad(i=e, h h),
$$

where $\Phi^{(i)}$ is the confinement potential energy for electron $(i=e)$ or heavy hole $(i=h h)$ given by $\Phi^{(i)}=\hbar^{2} \pi^{2} / 2 m_{i}^{*} L_{z}^{2}$ if an infinite barrier is assumed, and an additional energy shift $E_{\mathrm{QCSE}}$ is caused by the quantum confined Stark effect (QCSE) when perpendicular electric fields are applied. ${ }^{23}$ Unlike the first two cases, the emission energy shift due to the monolayer fluctuation of the QW width strongly depends on the perpendicular electric field. The perturbation calculation gives an analytical form of the $E_{\mathrm{QCSE}}{ }^{24}$ as

$$
E_{\mathrm{QCSE}}^{(i)}=-C\left(m_{i}^{*} e^{2} F^{2} L_{z}^{4}\right) / \hbar^{2},
$$

where $C$ is a numerical constant. This potential fluctuation can be expanded by the deviation from the averaged well width $\Delta L_{z}(\rho)=L_{z}(\rho)-\overline{L_{z}}$, where $\rho=(x, y)$ is the $2 \mathrm{D}$ coordinate in the QW plane and $\bar{L}_{z}$ is the spatially averaged width. By introducing the relative and center-of-mass coordinates, the center of the mass Schrödinger equation for $X^{-}$is given by

$$
\left\{\frac{\hbar^{2}}{2 M} \Delta_{R}+\Lambda(R)-\varepsilon_{\alpha}\right\} \psi_{\alpha}(R)=0,
$$

where $M=2 m_{e}^{*}+m_{h h}^{*}$ and $\Lambda$ is the potential for the center of mass. The variance of $\Lambda(R)$ due to the well-width fluctuation is averaged within the extent of the relative motion. Therefore, the broadening of the $X^{-}$emission spectrum can be derived using the similar method in Ref. 25 as

$$
\sigma^{2}=\left\langle\Delta L_{z}^{2}\right\rangle \frac{a_{i}^{2}}{A_{1}}\left(\frac{d W}{d L_{z}}\right)^{2},
$$

where $W$ is the sum of $W^{(i)}$ for the electron and hole that are annihilated in the PL emission process, $a_{i}$ refers to the island size determined by the growth process, and $A_{1}=\int d^{2} \rho \phi(\rho)^{4}$ is the statistically relevant area of the $X^{-}$. Assuming $2 \mathrm{D}$ exponential wave function $\phi(\rho)=\sqrt{2 / \pi a^{* 2}} \exp \left(-\rho / a^{*}\right)\left(a^{*}\right.$ is the trion radius) for the relative motion of $X^{-}, A_{1}$ is given as $\pi a^{* 2}$. By substituting Eq. (2), we finally obtain the relation

$$
\begin{aligned}
\sigma & =\frac{2\langle|\Delta L|\rangle}{\bar{L}} \frac{a_{i}}{\sqrt{\pi} a^{*}}\left(\left.2 E_{\mathrm{QCSE}}\right|_{L_{z}=\overline{L_{z}}}+\bar{\Phi}\right) \\
& =\frac{4\langle|\Delta L|\rangle}{\bar{L}} \frac{a_{i}}{\sqrt{\pi} a^{*}} \Delta \epsilon+\sigma_{0},
\end{aligned}
$$

where $\Delta \epsilon=\left.E_{\mathrm{QCSE}}\right|_{L_{z}=\overline{L_{z}}}$ is the energy shift caused by the QCSE and $\sigma_{0}=(2\langle|\Delta L|\rangle / \bar{L})\left(a_{i} / \sqrt{\pi} a^{*}\right) \bar{\Phi}$ represents the inhomogeneous linewidth at $F=0$. Note that the coefficient of $\Delta \epsilon$ in Eq. (5) is inversely proportional to $a^{*}$ and then the PL broadening $\sigma$ can be a good measure of the spatial extent of the $X^{-}$.

Now we shall see how the PL spectra change as a function of the electron density (see Supplemental Material ${ }^{26}$ ). Figure 1(b) shows the PL spectra at a fixed electron density plotted in the same panel for effective electric fields $F^{*}$ determined from the shift of PL emission energy. ${ }^{27}$ Two peaks observed at low electron density in Fig. 1(b) are the emission by $X_{0}$ and $X^{-}$. At higher electron density, in Figs. 1(c), 1(d), and 1(e), $X_{0}$ emission disappears and a single emission band of $X^{-}$or

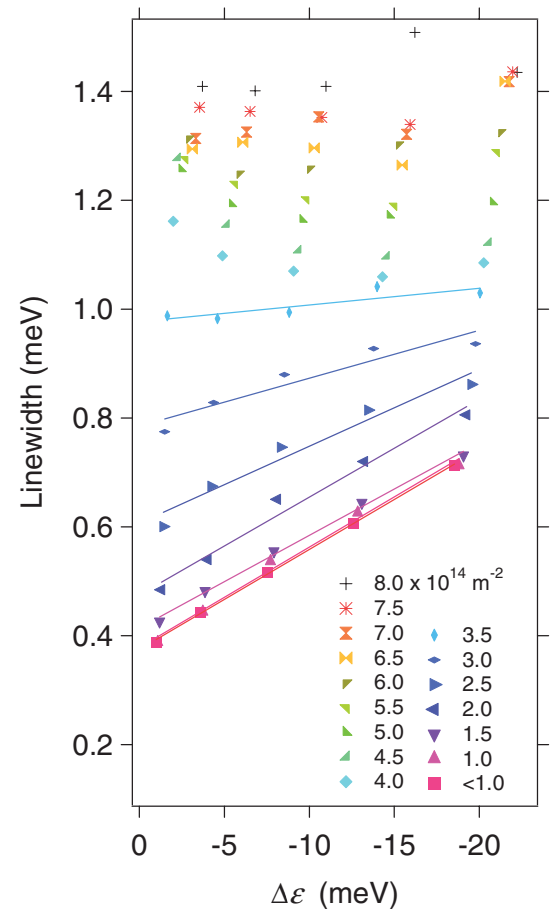

FIG. 2. (Color online) PL linewidth plotted as a function of PL energy shift $\Delta \epsilon$ for different electron densities.

2DEG-h remains. Figure 1(f) shows the $V_{f}$ dependence of the PL energy shift for $X^{-}$(or 2DEG-h) for fixed $n_{e}$. The PL emission energies largely shift when $V_{f}$ is applied regardless of the electron density, which implies that the perpendicular electric fields are effectively applied even at finite electron density and the observed energy shift is mostly due to the QCSE. Therefore, we reasonably assume $\Delta \epsilon=\epsilon-\epsilon(0)$ is the shift caused by the QSCE, where $\epsilon(0)=1.5265 \mathrm{eV}$ is the PL emission energy at $F=0$.

Figure 2 shows the full width at half maximum of $X^{-}$ (or 2DEG-h) spectrum $\Gamma$ plotted as a function of the emission energy shift $\Delta \epsilon$. In the low-density regime $n_{e}<4 \times 10^{14} \mathrm{~m}^{-2}$, the curves can be fit well by $\Gamma=\alpha \Delta \epsilon+\beta$. The change of $\Gamma$ with $\Delta \epsilon$ is due to the enhancement of the linewidth caused by the QCSE as derived in Eq. (5). At the lowest electron density, we obtained $\alpha=-1.83 \times 10^{-2}$ and $\beta=0.375 \mathrm{meV}$. Substituting the value of $\alpha$ and $\bar{\Phi}=\bar{\Phi}^{e}+\bar{\Phi}^{h h}=0.016 \mathrm{eV}$ in Eq. (5), we can estimate the inhomogeneous linewidth due to the monolayer fluctuation $\sigma_{0}\left(=\frac{\alpha}{2} \bar{\Phi}\right)=0.146 \mathrm{meV}$ and the residual linewidth including homogeneous linewidth $\beta-\sigma_{0}=0.229 \mathrm{meV}$ for $F=0$. The mixing line shape of inhomogeneous and homogeneous linewidth is consistent with the line shape of $X^{-}$at $F=0$ in the low-electron-density regime, which can be fit by the Voigt function with a mixed line shape of Gaussian and Lorentzian. In the higher-density regime $n_{e}>4 \times 10^{14} \mathrm{~m}^{-2}$, on the other hand, the linewidth does not change linearly against $\Delta \epsilon$. This implies that our simplified model Eq. (5) no longer holds for higher density and the PL spectrum is highly affected by the change in the electron states. Here, it is interesting that the linewidth decreases with increasing electric field in the intermediate regime $4 \times 10^{14}<n_{e}<5 \times 10^{14} \mathrm{~m}^{-2}$. With a further increase in the electron density, $n_{e}>6 \times 10^{14} \mathrm{~m}^{-2}$, the electric-field 


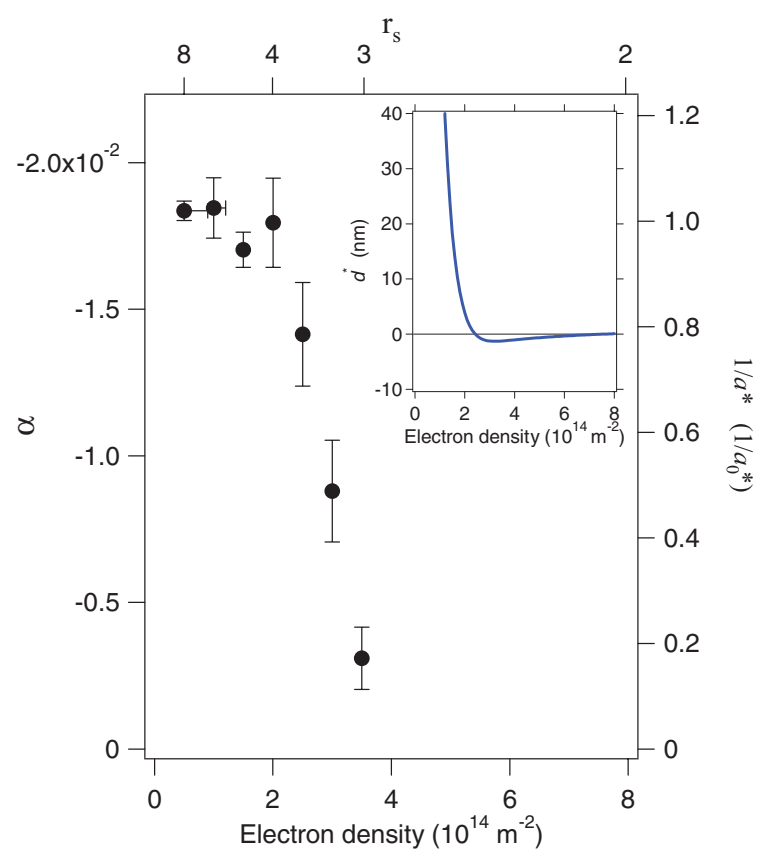

FIG. 3. (Color online) Electron-density dependence of $\alpha$, which is the slope of the PL linewidth against the PL energy shift. Corresponding trion radius $a^{*}$ is displayed on the right axis (see text). Top axis indicates $r_{s}$. Inset: screening length evaluated from the nonlinear screening theory using our sample parameters.

dependence of the PL linewidth becomes small where the PL spectrum shows a typical 2DEG-h signature with a Fermi energy and the band edge depending on the electron density.

The electron-density dependence of $\alpha$ is plotted in Fig. 3. This plot is the central result of the present paper. $|\alpha|$ (and then $\left.1 / a^{*}\right)$ is nearly constant for low density and suddenly drops at $n_{e}=2 \times 10^{14} \mathrm{~m}^{-2}$. This behavior can be explained in terms of the electron-density dependence of the screening length. The nonlinear screening theory predicts the screening length: ${ }^{28}$

$$
d^{*}=\frac{a_{B}}{4}+d_{\mathrm{ex}}+d_{\mathrm{cor}}+d_{\mathrm{dis}} .
$$

The first term is due to the single-particle density of states. The second and third terms correspond to the exchange interaction and correlation of the 2DEG. $d_{\mathrm{ex}}$ and $d_{\text {cor }}$ contribute negatively in low electron density. ${ }^{29}$ In the low-electron-density limit, a positive contribution of the disorder term $d_{\text {dis }}$ overcomes the negative contribution and $d^{*}$ increases rapidly as the electron density decreases. ${ }^{13,20}$ The screening length evaluated from the nonlinear screening theory for our sample is shown in the inset of Fig. 3. We see a clear correspondence between the drop of $\alpha$ at $n_{e}=2 \times 10^{14} \mathrm{~m}^{-2}$ and the rapid decrease of $d^{*}$ across the scale of $a_{0}^{*}$. For $n_{e}<2 \times 10^{14} \mathrm{~m}^{-2}, d^{*}$ is larger than $a^{*}$ and then $X^{-}$cannot be screened by the 2DEG and thus $a^{*} \sim a_{0}^{*}$, which explains the insensitivity of $\alpha$. When $d^{*}$ is decreased to the scale of $a_{0}^{*}$, the 2DEG starts screening the binding energy of $X^{-}$, resulting in the increase of $a^{*}$. From this result, we can estimate the electron-density dependence of the trion radius displayed on the right axis in Fig. 3 in the unit of $\frac{1}{a_{0}^{*}}$, where we assumed that $\alpha=-1.8 \times 10^{-2}$ corresponds to $a_{0}^{*}$. This analysis gives strong evidence that the crossover of the $X^{-}$ and $2 \mathrm{DEG}-\mathrm{h}$ state is mediated by the screening of the binding energy by the 2DEG in the density $2 \times 10^{14}<n_{e}<4 \times$ $10^{14} \mathrm{~m}^{-2}$. As shown in Fig. 3, this crossover occurs in a narrow range of the density parameter $r_{s}$ between 3 and 4 , which is much larger than the values previously reported for the Mott transition in a charge neutral system. ${ }^{4}$ The density regime $4 \times$ $10^{14}<n_{e}<5 \times 10^{14} \mathrm{~m}^{-2}$, where the $\Delta \epsilon$ dependence of $\Gamma$ shows negative dependence, corresponds to the regime where $d^{*}$ becomes negative. In this regime, the exchange interaction and correlation of the $2 \mathrm{DEG}$ is important. The observed negative slope in Fig. 2(b) may be associated with the negative compressibility of the highly correlated electron system. To the best of the authors' knowledge, no theoretical prediction is available for the optical properties in the negative compressibility regime, and this will be an interesting subject in the future.

In summary, we have measured the electric-field dependence of the PL linewidth as a function of the electron density. The slope $|\alpha|$ of the linewidth against the electric field reflects the spatial extent of the internal motion of $X^{-}$states. We found that $|\alpha|$ is almost constant at $n_{s}<2 \times 10^{14} \mathrm{~m}^{-2}$ and decreases at $2 \times 10^{14}<n_{s}<4 \times 10^{14} \mathrm{~m}^{-2}$. This clearly indicates that the extended 2DEG screens the binding energy of $X^{-}$and generates the crossover between $X^{-}$and 2DEG-h.

This work was partly supported by Grant-in-Aid for Scientific Research on Innovative Areas “DYCE” No. 20104005.
${ }^{1}$ N. F. Mott, Philos. Mag. 6, 287 (1961).

${ }^{2}$ C. F. Klingshirn, Semiconductor Optics, 2nd ed. (Springer, Berlin, 2005).

${ }^{3}$ T. Ando, M. Nakayama, and M. Hosoda, Phys. Rev. B 69, 165316 (2004).

${ }^{4}$ L. Kappei, J. Szczytko, F. Morier-Genoud, and B. Deveaud, Phys. Rev. Lett. 94, 147403 (2005).

${ }^{5}$ M. Yoshita, Y. Hayamizu, H. Akiyama, L. N. Pfeiffer, and K. W. West, Phys. Rev. B 74, 165332 (2006).

${ }^{6}$ T. Yoshioka and K. Asano, Phys. Rev. Lett. 107, 256403 (2011).

${ }^{7}$ M. A. Lampert, Phys. Rev. Lett. 1, 450 (1958).
${ }^{8}$ For reviews, see A. J. Shields, M. Pepper, D. A. Ritchie, and M. Y. Simmons, Adv. Phys. 44, 47 (1995); I. Bar-Joseph, Semicond. Sci. Technol. 20, R29 (2005), and references therein.

${ }^{9}$ Such as A. Nurmikko and A. Pinczuk, Phys. Today 46(6), 24 (1993).

${ }^{10}$ P. Hawrylak, Phys. Rev. B 44, 3821 (1991).

${ }^{11}$ G. Eytan, Y. Yayon, M. Rappaport, H. Shtrikman, and I. Bar-Joseph, Phys. Rev. Lett. 81, 1666 (1998).

${ }^{12}$ G. Yusa, H. Shtrikman, and I. Bar-Joseph, Phys. Rev. B 62, 15390 (2000).

${ }^{13}$ M. Yamaguchi, S. Nomura, T. Maruyama, S. Miyashita, Y. Hirayama, H. Tamura, and T. Akazaki, Phys. Rev. Lett. 101, 207401 (2008). 
${ }^{14}$ Y. Hirayama, K. Muraki, and T. Saku, Appl. Phys. Lett. 72, 1745 (1998).

${ }^{15}$ T. F. Rønnow, T. G. Pedersen, B. Partoens, and K. K. Berthelsen, Phys. Rev. B 84, 035316 (2011).

${ }^{16}$ L. C. O. Dacal and J. A. Brum, Phys. Rev. B 65, 115324 (2002).

${ }^{17}$ S. Nomura, M. Yamaguchi, T. Akazaki, H. Tamura, T. Maruyama, S. Miyashita, and Y. Hirayama, Phys. Rev. B 76, 201306(R) (2007).

${ }^{18}$ M. Yamaguchi, S. Nomura, K. Miyakoshi, H. Tamura, T. Akazaki, and H. Takayanagi, J. Appl. Phys. 100, 113523 (2006).

${ }^{19}$ A. L. Efros, Solid State Commun. 70, 253 (1989).

${ }^{20}$ M. M. Fogler, Phys. Rev. B 69, 121409(R) (2004).

${ }^{21}$ L. V. Butov, V. D. Kulakovskii, T. G. Andersson, and Z. G. Chen, Phys. Rev. B 42, 9472 (1990).

${ }^{22}$ V. V. Solovyev and I. V. Kukushkin, Phys. Rev. B 79, 233306 (2009).

${ }^{23}$ D. A. Miller et al., Phys. Rev. Lett. 53, 2173 (1984).
${ }^{24}$ G. Bastard, E. E. Mendez, L. L. Chang, and L. Esaki, Phys. Rev. B 28, 3241 (1983).

${ }^{25}$ R. Zimmermann, Phys. Status Solidi B 173, 129 (1992).

${ }^{26}$ See Supplemental Material at http://link.aps.org/supplemental/ 10.1103/PhysRevB.87.081310 for additional experimental results of (1) currents flowing through the front and gate bias lines, (2) photoluminescence spectrum in magnetic fields, and (3) capacitance between the QW and back gate.

${ }^{27}$ Effective electric field for a PL emission energy $\epsilon$ at a finite electron density $n_{e}>0$ is evaluated from the equation $F^{*}=f^{-1}(\Delta \epsilon)$ where $\Delta \epsilon=\epsilon-1.5265 \mathrm{eV}$ and the function $f$ is determined by $\Delta \epsilon=$ $f(F)$ at zero electron density $n_{e}=0$.

${ }^{28}$ G. Allison et al., Phys. Rev. Lett. 96, 216407 (2006).

${ }^{29}$ J. P. Eisenstein, L. N. Pfeiffer, and K. W. West, Phys. Rev. B 50, 1760 (1994). 